\title{
One-shot focusing using the entropy as a merit function
}

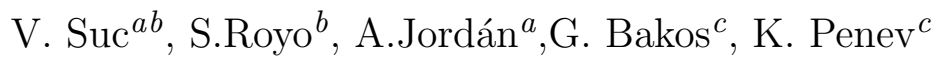 \\ ${ }^{a}$ Departamento de Astronomía y Astrofísica \& Centro de Astroingeniería, \\ Pontifícia Universidad Católica de Chile, Vicuña Mackenna 4860, 7820436 Macul, Santiago, \\ Chile; \\ ${ }^{b}$ Centre for Sensors, Instrumentation and Systems Development, \\ Universitat Politécnica de Catalunya(UPC-CD6), Rambla Sant Nebridi 10 E08222 Terrassa, \\ Spain \\ ${ }^{c}$ Department of Astrophysical Sciences, Princeton University, NJ, USA
}

\begin{abstract}
We present a method for measuring focus aberrations on wide field telescopes based on an entropy analysis of a single image. First, we calibrate the system using the evolution of the entropy as a function of the position in the field and the focuser position. This gives us a model defining the tilt of the sensor and the field curvature. Then, using a single image at a given focuser position in which the mean defocus is unknown, we can compute the position where the focuser must be set in order to minimize the focus aberration over the whole field.
\end{abstract}

Keywords: Image metrics, sensorless adaptive optics, one-shot focusing

\section{INTRODUCTION}

One of the most basic features one often desires in telescope operations is to keep the system at or as near as possible to an optical focus position. Historically this was done by adjusting the focus manually such that the narrowest point spread function possible was achieved as judged by the eye on stellar images. Large modern telescopes rely on automated systems such as those defined by, e.g., Mc Leod et al. (1) or Kuehn (2).

In this paper we present an algorithm to find the best focus position of a telescope delivering wide-field astronomical images in a non-iterative way. Debarre et al (3) (4) (5) showed that given $N+1$ measurements of some merit function of a system aberrated by $N$ offsets applied on $N$ different optical modes, it was possible to minimize the aberration of these $N$ modes. In our case we want to minimize a single optical aberration on a wide field image which is defocus. In order to be able to find the best mean value of the defocus using a single image, we consider the field curvature as a radial function of the distance to the optical center and proceed in 4 steps:

- First, we calibrate the field curvature, sensor tilt, and the position of the optical center using a set of images which are affected by different amounts of known mean defocus.

- In step two, we determine a model function that allows us to associate an absolute defocus value with a set of values of a given image quality metric.

- The third step consists in measuring the value of the image quality metric chosen in step 2 in different parts of the image.

- Finally, a minimization algorithm will find the mean defocus value which best fits the data obtained in step 3 using the model obtained in step 2.

Steps 1 and 2 can be done a single time for the instrument lifetime (as long as it is stable and it is not intervened), while steps 3 and 4 will be run every time we want to compute the best focus position as determined by a single image. In this work we use data of focus series taken with four telescopes of the HAT-South project(6) to illustrate the procedure outlined above. The four HAT-South telescopes are based on the same optical design but may be affected by different alignments of the sensors relative to the corresponding optical axes. We show 
that the local entropy of a subimage around the detected stars is the best image metric for our application, rather than other image metrics more usual in the definition of astronomical image quality, such as full-width at half maximum. Finally, we briefly discuss how stable is the proposed technique under variations of the model such as, e.g., variations in the sensor tilt which can arise due to instrumental flexure. The four steps we have described will be analyzed in detail along the text.

\section{STEP 1: CALIBRATION ALGORITHM}

The first step in the procedure is the calibration of the field curvature, sensor tilt, and the position of the optical centre, using a set of images with known mean defocus. The calibration algorithm we propose has been tested on a focus sequence of thirty images taken with the HS1.4 telescope of the Hat-South Las Campanas Observatory (LCO) station (60 images in total). Every image was taken with the focuser in a different known position. We thus know the absolute focus position in coding units of stepper motors, and we want to find the parameters $z, \vec{O}, \vec{m}, \vec{t}$ which are defined as:

- Defocus offset $z$ (offset from the optical focus position)

- Position of the field center $\vec{O}=\left(O_{x}, O_{y}\right)$

- The field curvature, which can be expressed as a quadratic function from the distance to the field center, defined by the scalars $\vec{m}=\left(m_{1}, m_{2}\right)$

- Detector tilt, which is defined by a linear funcion defined by the parameter $\vec{t}=\left(t_{x}, t_{y}\right)$

The procedure to obtain these parameters is described next.

\subsection{Single image analysis}

For every image we measure a given number of metric values around every detected star. In order to perform star detection in our frames we use SExtractor (7). The metrics we extract for each star are

- Full width at half-maximum (FWHM).

- Stellar ellipticity.

- Entropy of a box around the star.

Entropy is defined according to Equation 1, where $P$ is the normalized binned histogram of the values in a 10x10 pixel $^{2}$ box around the star:

$$
e=\sum_{i=0}^{\operatorname{len}(P)} P_{i} \log \left(P_{i}\right)
$$

The measured values for each star are then smoothed using a 10x10 grid representing the full field of view. Every box of the mesh has an associated value of the metric at its center, which is the mean of all values of the sources present in the considered box. The value of the metric at an arbitrary position in the box is obtained by interrelating from the grid. An example of a calculated entropy map in a single image is shown in Fig 1 . An extended analysis of several images affected by different values of mean defocus shows that images before focus tend to be present better results close to the optical center rather than on the edges, while images taken behind the focus tend to present better results on the edges rather than on the center of the field. This assymetry is due to the effect of field curvature, and as a result, a detailed analysis of any defocus image that can measure this assymmetry should in principle be sufficient to determine on which side of the focus we are and how far we are from the best focus position. Thus, the local behavior of entropy becomes a key issue in the process and deserves further attention. 


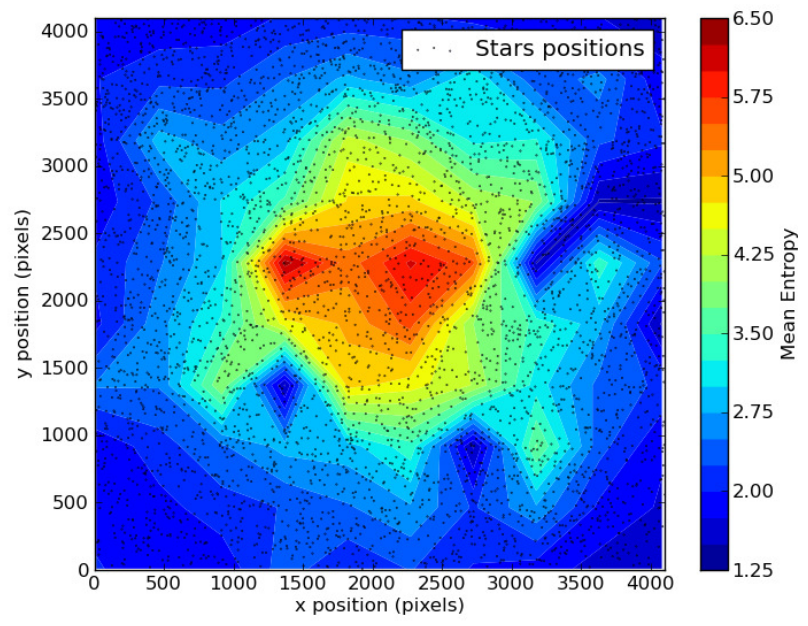

Figure 1. Entropy measured on one image.

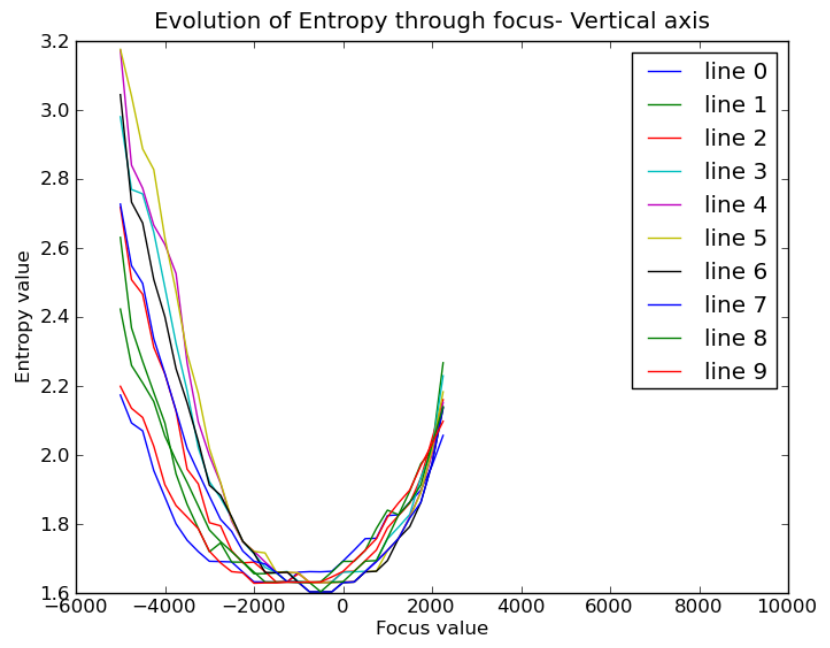

Figure 2. Evolution of the mean entropy on 10 zones of the field as a function of the focus position.

\subsection{Local evolution of entropy}

When we divide a given image in a $10 \times 10$ zone map, we can probe the evolution of the mean entropy (or any other image metric) as the defocus is changed to evaluate which is its behavior. The graph shown in Fig 2 shows the evolution of entropy as a function of focus position for 10 zones in the HAT-South images series at different focus positions. Entropy consistently behaves as smoothly varying with focus change, and it obtains its minimum value at the best focus position for each zone considered. Clearly, however, this best focus position is not the same for all the zones of the images, but the behaviour of the entropy as a smooth, derivable function of focuser position is equivalent from zone to zone.

Next step will be the development of a intrumental model of the focusing of the telescope. In order to further characterize the variation of a given metric on a given zone we choose to fit Gaussian functions to the entropy values at each zone. 


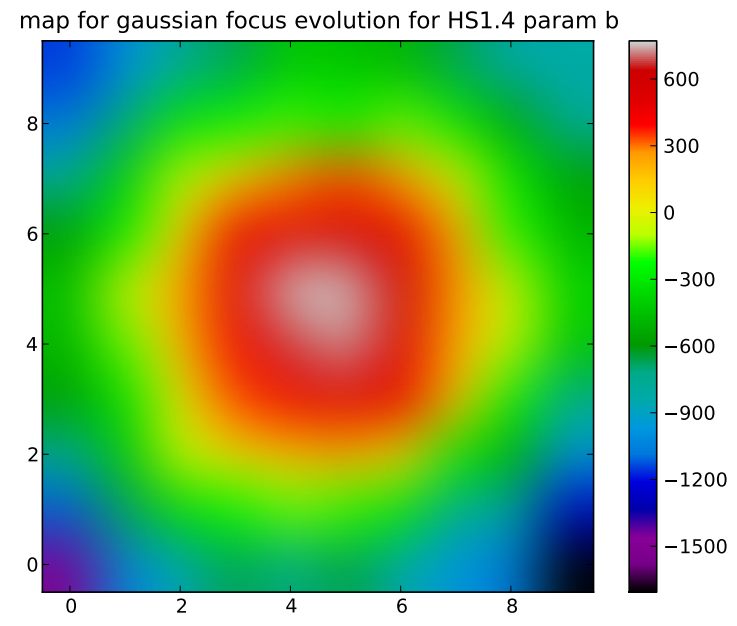

Figure 3. $b$ value (mean of the Gaussian fit) over the field of HS1.4

\subsection{Optical instrumental model}

We then fit a Gaussian function of the form

$$
f(x)=a e^{-\frac{(x-b)^{2}}{c}}+c
$$

to the variation of a given metric in each zone of the image. We then can extract from the fit the value of the Gaussian mean $b$, and plot its value over the entire field of view of the image. The best fit value of $b$ over the field obtained with our set of focus images for HS1.4 is shown Fig 3 .

The map obtained from this figure gives us the value of the best focus as a function of the position in the field $(x, y)$ calculated out of the entropy value. From this information we can estimate the parameters $\{z, \vec{O}, \vec{m}, \vec{t}\}$ defined previously. In order to achieve this, we use the map of $b$ values obtained to fit the following model:

$$
b=z+t_{x}\left(x-O_{x}\right)+t_{y}\left(y-O_{y}\right)+m_{1} r_{(x, y)}+m_{2} r^{2}(x, y)
$$

where $r^{2} \equiv\left(x-O_{x}\right)^{2}+\left(y-O_{y}\right)^{2}$. The multidimensional model for HS1.4 and its residuals is shown in Fig. 4 , where we see that a reasonable quality fit is obtained enabling all parameters to be calculated, including the best focus position for the image (in this case, $264.99 \mathrm{~mm}$ ). A better fit could have of course been obtained with a 3rd or higher order model, but this would have made the fitting and later evaluation processes slower and, as we will show in the following, a second order model proves to be accurate enough for our purposes.

Thus, a consistent model of the focus position of the telescope based on the analysis of the evolution of the entropy function has been obtained. the model allows the determination of the best focus for the image plus the tilt, vertex position and field curvature.

\section{STEP2: MODEL OF THE BEHAVIOUR OF ENTROPY AS A FUNCTION OF ABSOLUTE DEFOCUS}

\subsection{Analysis of different image metrics}

At this point we have a model of our telescope which, out of its images, enables the characterization of field curvature, CCD tilt and position of the optical axis. This allows us to predict the best focus position $b$ for each 

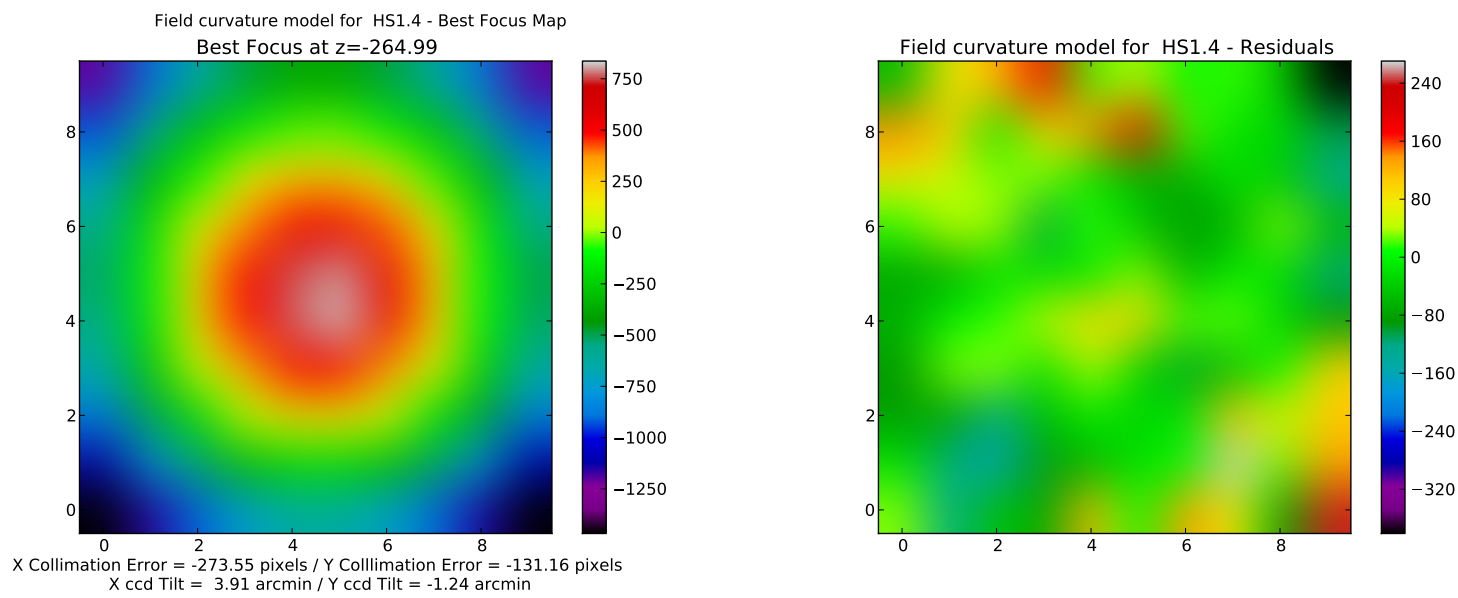

Figure 4. Optical $z O m t$ model and residuals

star on a given image out of its position in the field. We can then plot the evolution of any given metric measured on a per star basis as a function of its absolute defocus, i.e. the value of its defocus relative to the best focus position at the position of the source. The best metric will be the one which shows a nearly constant behavior across the whole field of view, as far as this will allow to use this single function to model the effects of defocus on our instrument. We carried out this exercise for the three image metrics which were mentioned above, namely FWHM, ellipticity and entropy. The results are shown in Fig 5,6 and Fig 7. It is clear from the figures that entropy is a significantly more stable metric at all positions of the field of view, in the sense that its behavior as a function of absolute defocus is more homogeneous across the field, making it the best metric for our purposes and enabling to predict the best focus position of the telescope out of single images.

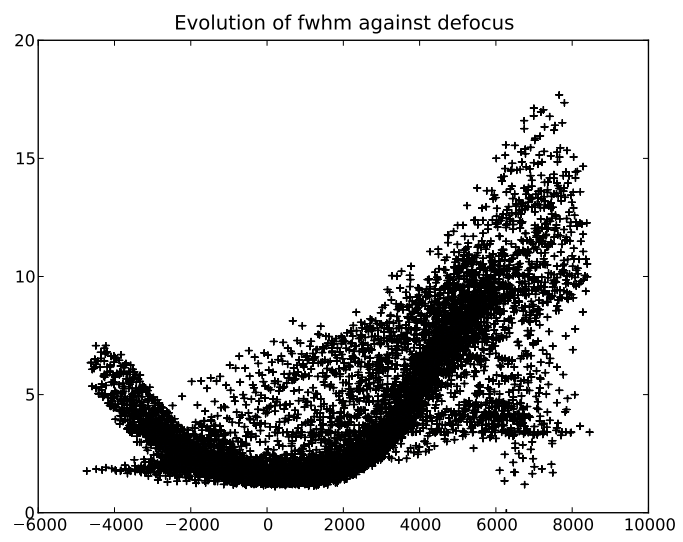

Figure 5. FWHM metric values as a function of defocus.

\subsection{Entropy behavioral model}

Once the entroipy has been fixed as the optimal image metrics for our applications, it will now be simple to define a behavioral model which will associate an entropy value to a known absolute defocus. This model is based on a functional fitting of the entropy measurements shown in Fig. 7. When testing quadratic, cubic and gaussian functions as basis functions for the fit the results showed a very limited quality fit to the set of points, so we turned into non-parametric functions to model entropy. Our first trial was a 1-dimensional smoothed spline. We can see this model overlaid on the experimental data points in Fig. 8. 


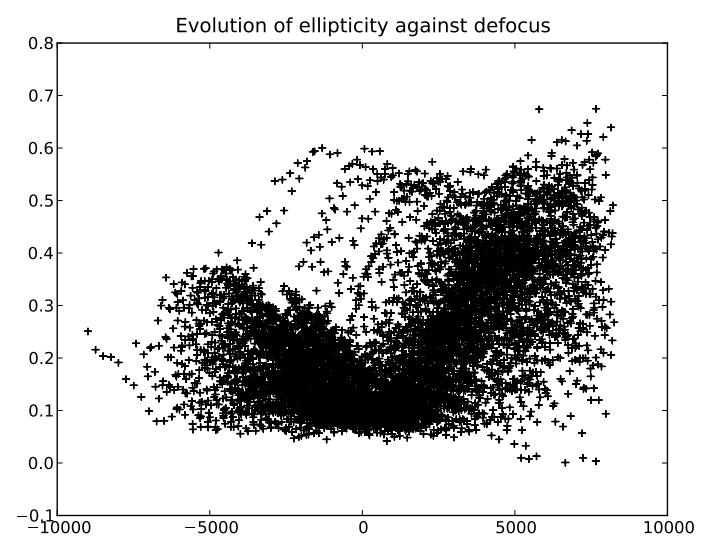

Figure 6. Ellipticity metric values as a function of defocus.

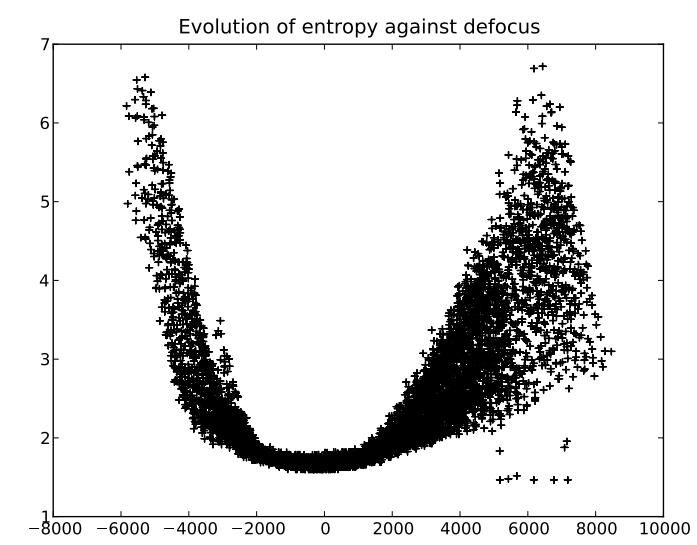

Figure 7. Entropy metric values as a function of defocus

If the evolution of entropy is plotted in a 3D plot including absolute defocus and distance to the centre, it may be seen how the behavior of the focal point has a certain dependence on the distance to the entropy optical axis(see Fig. 9). In order to further strengthen the model and to make it more accurate, we tried also the fit to a 2-dimensional model based on a smoothed b-spline. This model is shown in Fig. 9. The residuals of both the $1 \mathrm{D}$ and the 2D fits may be seen in Fig. 10, with the logical better fit in the case of the 2D spline.

\section{STEP3: FOCUS DETERMINATION}

Once the optical model and the behavioral model have been constructed as described in the previous Sections, we are now able to associate the modelled entropy in any position of the field with an absolute defocus value so optimal focusing is consistently attained. We can now use this information to determine the mean defocus of an image for which this quantity is unknown. Given such image, we first find the stars present in the image and compute their associated entropy, generating a vector of measured entropies for the image, $\vec{e}=\left(e_{1}, \ldots, e_{n}\right)$. Using the model of entropies we have developed $\vec{m}(b)$, we can find the value of absolute defocus which minimizes the amount of defocus of all of them

$$
X^{2}(b)=\sum_{i}\left(e_{i}-m_{i}(b)\right)^{2}
$$




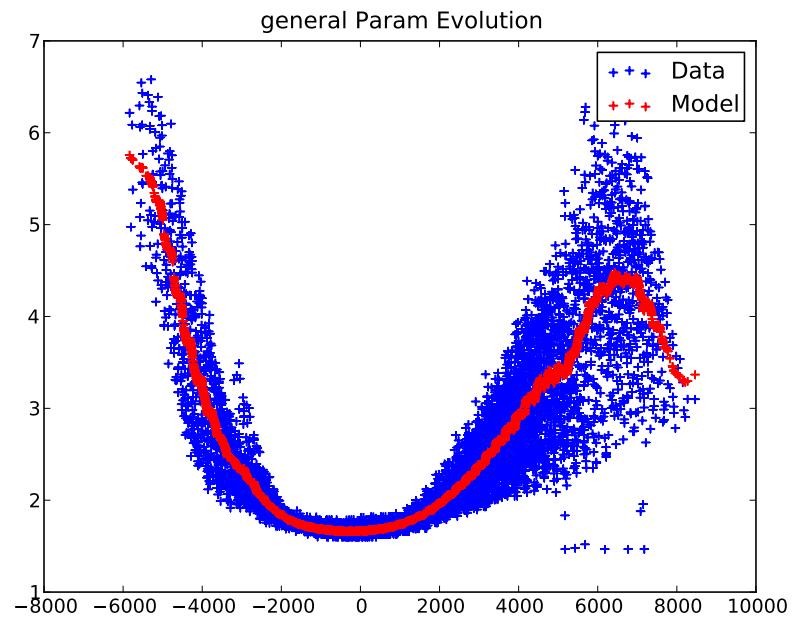

Figure 8. Entropy value against defocus (points) and fit to a 1D smoothed spline (line)
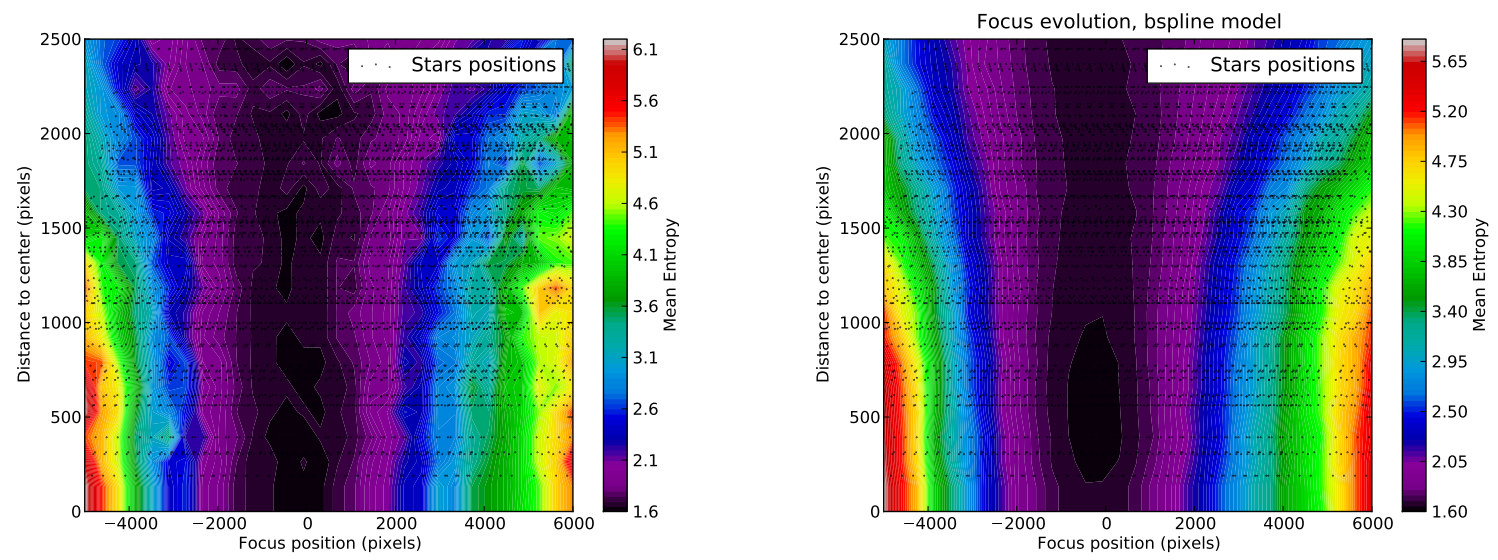

Figure 9. Entropy value against defocus (points), and fit to a $2 \mathrm{~d}$ smoothed spline (model)

and that gives us directly the best focus position. Figure 11 shows the evolution of $\|\vec{e}-\vec{m}(b)\|$ as a function of $b$ for an image whose actual defocus is -6000 units. Figure 12 shows the same for an image with 1000 units of defocus.

We tried several algorithms to find the best focus by minimizing $X^{2}$ (Levemberg-Marquardt, simplex and absolute minimum search over an interval). It so happens that the error function has several minima around the absolute best focus, which makes the convergence with local search algorithms not very stable. The most robust solution was obtained generating a entropy vector for a grid of focuser positions inside an interval around a guess position. The algorithm returns the absolute minimum norm of residuals as the absolute best focus and a local refinement is done by interpolation around this position.

The stability of the algorithm was tested by computing defocus values for images on which the tilt and collimation were different from the one determined in the calibration model. It appears that for typical tilt errors of a few arcminutes the focusing algorithms converges to a similar position. This confirms the robustness of the method against mechanical flexures of the telescopes. 

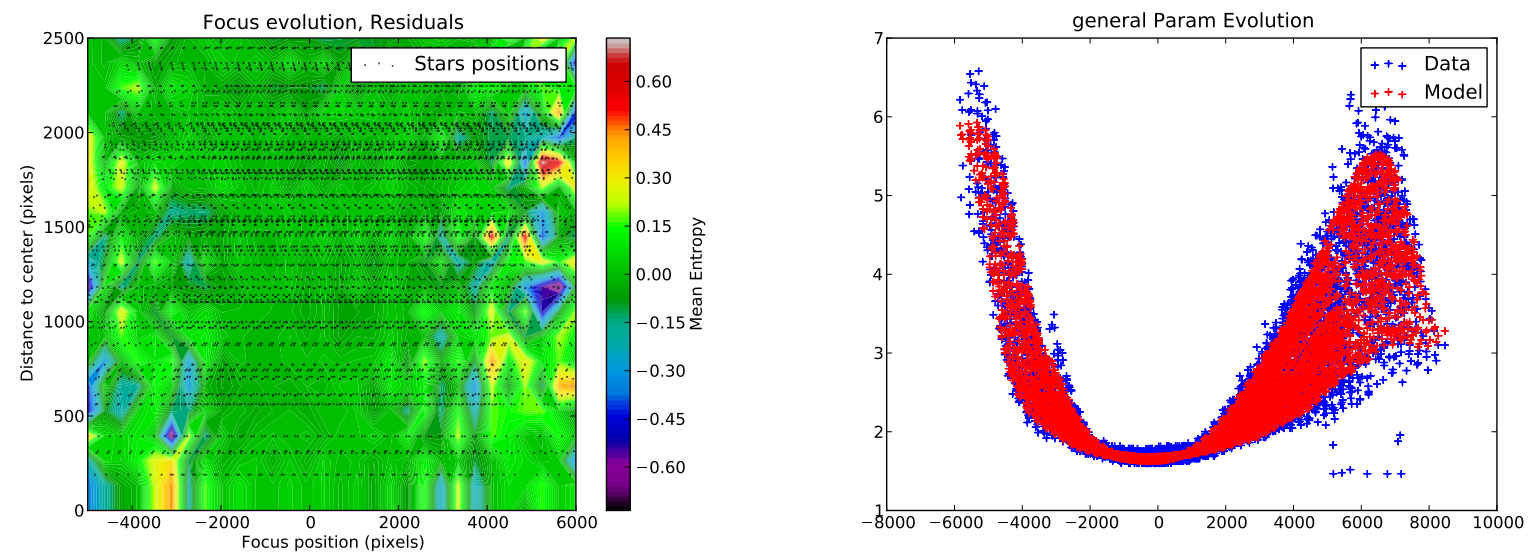

Figure 10. Entropy against defocus, $2 d$ residuals in $2 d$ and $1 d$

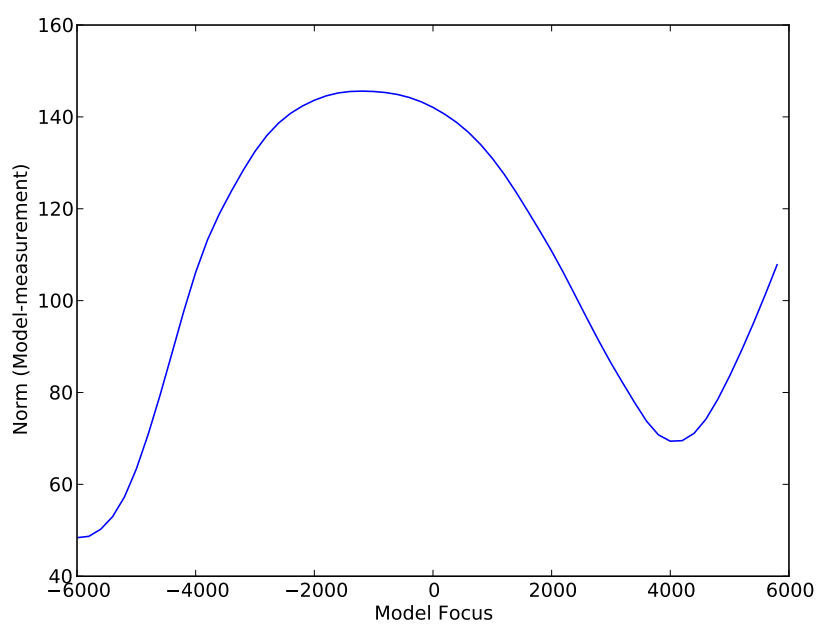

Figure 11. mean residuals for an image 6000units before focus

\section{DISCUSSION}

We have presented a new technique of one shot focusing using an entropy based merit function. Our results show that this technique can be more robust compared to usual techniques based on the use of fwhm or ellipticity as a metric of image quality.

This stability can be explained by the shape-independent nature of the entropy function compared to the others metrics. For both ellipticity and fwhm, the metric is computed form a $2 \mathrm{~d}$ gaussian fit of the star. This fit can become unstable as soon as the star is affected by a significant amount of defocus or coma since the shape to be fitted is not approximately gaussian anymore.

As a result, the focusing technique we have described can become a useful tool when applied to fast ratio or wide field telescopes. In these configurations, the focusing precision is critical to keep gaussian shaped images over the field as the coma affecting defocused stars near the edge of the field may prevent classical technique from converging properly.

We are currently finishing a control program capable of analyzing in realtime images taken in a series and compute the focus correction to be applied according to the focus position recorded in the image header. The computed correction can feed either directly to a focuser peripheral or to a telescope control scheduler. 


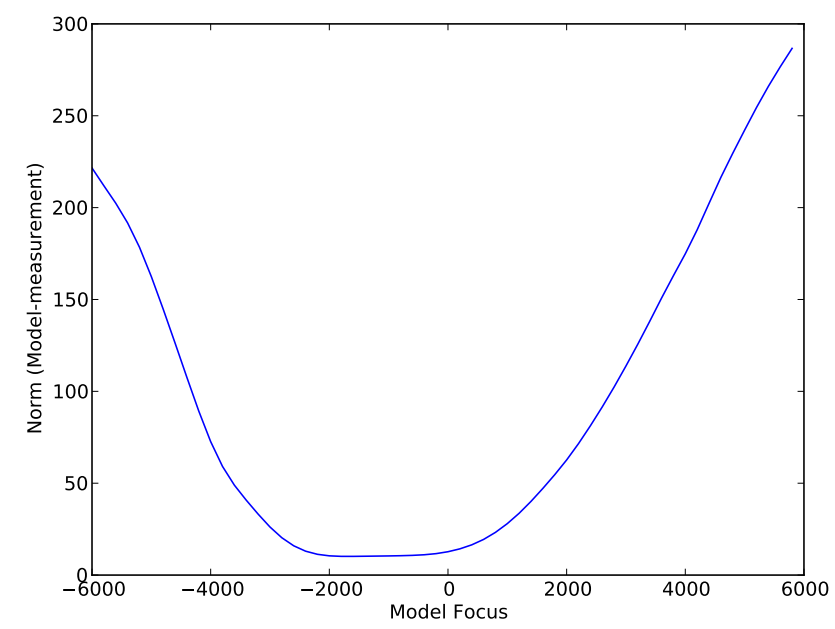

Figure 12. mean residuals for an image 1000 units before focus

\section{ACKNOWLEDGMENTS}

The authors would like to acknowledge the contribution of ACC1Ó (Catalan Agency for Competitiveness) for project VALTEC09-1-0063, of MINECO (Spanish Ministry for Economy and Competitiveness) for project DPI2011-25525, of the Basal Center for Astrophysics and Applied Technologies for the project PFB06, of the Project Anillo ACT-86, of FONDECYT for the project Regular 1095213, and of the Fondap Center for Astrophysics for the project Nr. 15010003.

\section{References}

[1] SAO, ed., [Megacam: Paving the focal plane of MMT with silicon], Proc. SPIE (July 1998).

[2] Kuehn, K. and Hupe, R., "Real-Time Analysis of Large Astronomical Images," ArXiv e-prints (Mar. 2012).

[3] Debarre, D., Booth, M. J., and Wilson, T., "Image based adaptive optics through optimisation of low spatial frequencies," Opt. Express 15(13), 8176-8190 (2007).

[4] Booth, M., "Wave front sensor-less adaptive optics: a model-based approach using sphere packings," Opt. Express 14(4), 1339-1352 (2006).

[5] Booth, M. J., "Wavefront sensorless adaptive optics for large aberrations," Opt. Lett. 32(1), 5-7 (2007).

[6] Bakos, G., Afonso, C., Henning, T., Jordán, A., Holman, M., Noyes, R. W., Sackett, P. D., Sasselov, D., Kovács, G., Csubry, Z., and Pál, A., "Hat-south: a global network of southern hemisphere automated telescopes to detect transiting exoplanets," Cambridge Univ. Press (2009).

[7] E. Bertin and S. Arnouts, "Sextractor: Software for source extraction," Astron. Astrophys. Suppl. Ser. 117(2), 393-404 (1996). 